
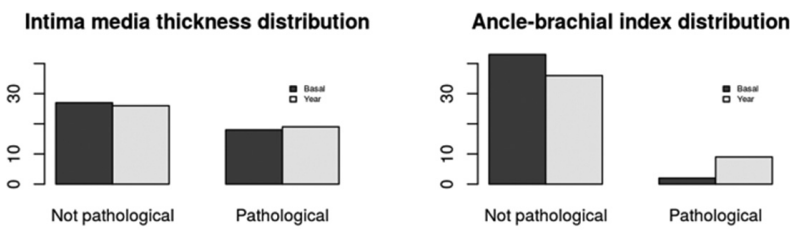

Endothelial disfunction distribution

Atheromatous plaque distribution
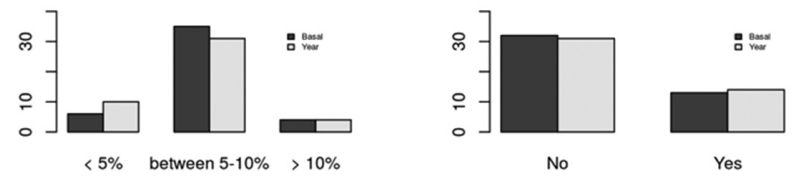

Yes

dose ultraviolet A-1 $\left(50 \mathrm{~J} / \mathrm{cm}^{2}\right)$ versus low-dose ultraviolet A-1 $\left(20 \mathrm{~J} / \mathrm{cm}^{2}\right)$ phototherapy versus narrowband UVB.

We are uncertain regarding adverse effects of interventions as the certainty of the evidence was very low. However, participants reported marked pain and pruritus during fractional carbon dioxide laser therapy, and had mild tanning after ultraviolet A-1 phototherapy.

Conclusions: There is a lack of high-certainty evidence for the treatment of morphea, and the effectiveness and safety of the interventions are unclear. Low-certainty evidence supports the effectiveness of oral methotrexate plus oral prednisone for treating juvenile morphea. More studies are necessary to assess the effectiveness and safety of interventions for morphea.

Acknowledgements: This abstract is based on a draft and pre-peer review version of a Cochrane Review. Upon completion and approval, the final version is expected to be published in the Cochrane Database of Systematic Reviews (www.cochranelibrary.com).

Disclosure of Interest: None declared

DOI: 10.1136/annrheumdis-2018-eular.6047

Abstract AB0772 - Figure 1

Conclusions: In our study, we observed the significant variation in $A B I$ in one year, this may be due to the fact that this measurement has a high sensitivity for the detection of early peripheral arterial disease, in those patients who have not manifested signs and symptoms of arterial disease due to more evolved time of evolution.

Acknowledgements: Thank you Casandra Jimenez for her help with the vascular database compilation

Disclosure of Interest: None declared

DOI: 10.1136/annrheumdis-2018-eular.3998

\title{
AB0773 INTERVENTIONS FOR MORPHEA: A COCHRANE SYSTEMATIC REVIEW
}

J.V. de Albuquerque ${ }^{1}$, B.NG. Andriolo ${ }^{1}$, M.RA. Vasconcellos2 ${ }^{2}$, V.T. Civile ${ }^{1}$, A. Lyddiatt ${ }^{3}$, V.FM. Trevisani ${ }^{1} .{ }^{1}$ Cochrane Brazil, Centro de Estudos de Saúde Baseada em Evidências e Avaliação Tecnológica em Saúde; ${ }^{2}$ Universidade Federal de São Paulo, São Paulo, Brazil; ${ }^{3}$ Cochrane Skin Group, The University of Nottingham, Nottingham, UK

Background: Morphea is a chronic inflammatory and fibrosing disorder usually limited to the skin and underlying tissues. It is an immune-mediated disease in which excess synthesis and deposition of collagen in the skin and connective tissues results in hardened cutaneous areas.

Objectives: To assess the effectiveness and safety of treatments for individuals with any form of morphea.

Methods: We searched the following databases up to March 2017: the Cochrane Skin Specialised Register, CENTRAL, MEDLINE, Embase, LILACS, and five trials registry databases. We checked the reference lists of included studies for further references to relevant randomised controlled trials.

We included randomised controlled trials assessing the effects of topical, intralesional, or systemic treatments for morphea (isolated or combined).

Two authors independently assessed study eligibility, extracted data, assessed risk of bias and performed analyses. A third author settled any disagreements.

Results: We included 13 trials, totalling 426 participants. There were both juvenile and adult participants (mostly women). The majority had limited morphea, followed by linear morphea.

The studies evaluated heterogenous therapies for morphea, covering a wide range of comparisons. Thus, we could not pool data from the studies in a metaanalysis. Six studies investigated topical medications, two evaluated intralesional medications, and five investigated systemic medications.

Regarding our primary outcome global improvement of disease activity or damage:

- The number of juvenile participants with a significant clinical response was higher with oral methotrexate plus oral prednisone than with placebo plus oral prednisone (RR 2.31, 95\% Cl 1.20 to 4.45, after the 12 month treatment; NNT 3; lowcertainty evidence);

- We are uncertain whether fractional carbon dioxide laser and the combination of acupuncture, hot herbal compress and moxibustion plus Centella triterpenes tablets and vitamin E may reduce this outcome, as the certainty of the evidence was very low;

- We found no differences in the MSS score between the following comparisons (very low-certainty evidence): oral hydroxychloroquine plus topical corticosteroid versus oral methotrexate plus folic acid and topical corticosteroid; and medium-

\section{$\mathrm{AB} 0774$ \\ IMPACT OF STANDARDISED EDUCATION PROGRAM ON THE ACCURACY OF MODIFIED RODNAN SKIN SCORING IN PATIENTS WITH SYSTEMIC SCLEROSIS}

J.W. Park ${ }^{1}$, J.-B. Jun ${ }^{2}$, L. Czirják ${ }^{3}$, Y. Allanore ${ }^{4}$, M. Matucci-Cerinic ${ }^{5}$, E.B. Lee ${ }^{6}$ ${ }^{1}$ Division of Rheumatology, Department of Intermal Medicine, Seoul National University College of Medicine; ${ }^{2}$ Department of Rheumatology, Hanyang University Hospital for Rheumatic Diseases, Seoul, Korea, Republic of Ireland; ${ }^{3}$ Department of Rheumatology and Immunology of Medical Centre, University of Pécs, Hungary; ${ }^{4}$ Paris Descartes University and Cochin Hospital, AP-HP, Paris, France;

${ }^{5}$ Department of Experimental and Clinical Medicine, University of Florence, Florence, Italy; ${ }^{6}$ Division of Rheumatology, Department of Internal Medicine, Seoul National University College of Medicine, Seoul, Korea, Republic of Ireland

Background: Modified Rodnan skin score (mRSS) has been used as not only a primary outcome in many clinical trials, but also as an important surrogate marker of disease activity in patients with systemic sclerosis (SSc). Therefore, establishment of well-organised training program of mRSS is essential for the proper management of patients. Recently, Scleroderma Clinical Trials Consortium and the World Scleroderma Foundation published the recommendation for 2-phase mRSS training and emphasised assessing scoring accuracy after the training. Objectives: To investigate the effect of modified Rodnan skin scoring (mRSS) education on improving its accuracy

Methods: Ten rheumatologists (6 professors and 4 fellows) received an education program composed of video education and live demonstration by master instructor (Marco Matucci-Cerinic) at (Seoul in June, 2017). Physicians measured mRSS of 8 patients with SSc 1) before the education, 2) after the video education and 3) after live demonstration without any clinical information of the patients. Accuracy of skin scoring was estimated by the difference from the pre-defined gold-standard score measured by master instructor. Change in accuracy of mRSS during the education course was analysed using linear mixed model. Intraobserver reliability of the mRSS and its change was assessed by intraclass correlation coefficient (ICC).

Abstract AB0774 - Table 1. Multivariable analysis indicating effect of the education program on the accuracy of modified Rodnan skin scoring

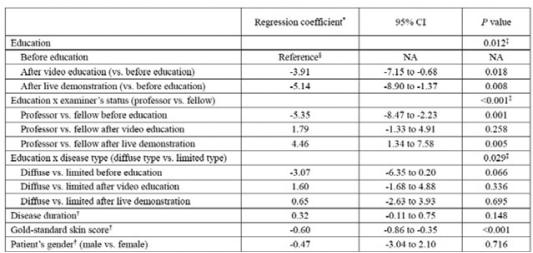

*, Dependent variable $=($ Examiner's skin score - gold-standard skin score $)$. Regression coefficient indicates the difference in dependent variable as compared with reference.

$\uparrow$, This clinical factor consistently influence the dependent variable irrespective of education course.

$\$$, indicates $P$ value for type 3 fixed effect

§, (Physician's skin score - gold-standard skin score) $(95 \% \mathrm{Cl})$ was estimated as 7.66 (6.03 to 9.29 ) before the education.

Results: The number of SSc patients ever experienced by each physician was significantly higher in the professors than fellows but the number of mRSS ever performed was comparable between the two groups. Median (IQR) skin score 
measured by master instructor was 10.5 (9.0). Mean (SD) difference between skin scores by physicians and master instructor was 7.7 (9.5) units. In the univariable analysis, video education significantly reduced the difference from the gold-standard score $(\beta=-1.96,95 \% \mathrm{Cl}-3.83$ to -0.10$)$ whereas live demonstration did not showed additional enhancement in scoring skill. Effect of education program was significantly different according to the physician's status and patient's disease type (diffuse vs. limited). In addition, male patient, shorter disease duration and higher gold-standard skin score was associated with more accurate skin scoring irrespective of the education. In the multivariable analysis where above clinical factors were adjusted, video education also led to significantly accurate skin scoring (table 1). When the educational effect was stratified by individual site of examination, face and distal extremities showed greater enhancement of scoring accuracy whereas difference from gold-standard score in proximal extremities was rather increased. ICC of physicians' skin scores was acceptable over all scoring times ( 0.63 to 0.88 ) but was not significantly changed after the education. Conclusions: The mRSS education program can significantly enhance the accuracy of $\mathrm{mRSS}$, which is mainly achieved by video education.

\section{REFERENCE:}

[1] Khanna D, Furst DE, Clements PJ, et al. Standardization of the modified Rodnan skin score for use in clinical trials of systemic sclerosis. J Scleroderma Relat Disord 2017;2(1):11-18. doi: 10.5301/jsrd.5000231

Disclosure of Interest: None declared

DOI: 10.1136/annrheumdis-2018-eular.3837

\section{AB0775 CHARACTERISTICS OF PATIENTS WITH SCLERODERMA (SSC) TREATED WITH VARIOUS DRUGS IN THE CLINICAL ASSESSMENT AND TGF B AND IL13 CONCENTRATION IN COMPARISON TO THE HEALTHY GROUP}

K. Romanowska-Próchnicka ${ }^{1,2}$, A. Wajda ${ }^{3}$, A. Paradowska-Gorycka ${ }^{3}$, M. Walczyk², M. Marecka², J. Trochimiuk², D. Szukiewicz ${ }^{1}$, M. Olesińska ${ }^{2}$. ${ }^{1}$ Department of General and Experimental Pathology, CEPT laboratory, Medical University of Warsaw, ${ }^{2}$ Department and Polyclinic of Systemic Connective Tissue Diseases; ${ }^{3}$ Department of Molecular Biology, National Institute of Geriatrics, Rheumatology and Rehabilitation, Warsaw, Poland

Background: Scleroderma (SSc) is a rare multisystem chronic disease the treatment of which is still challenging. Until now, there is no effective therapy that can modify the overall disease course. However, the main aim of Ssc treatment is directed toward managing organs involvement and providing symptomatic relief. Effective drug therapy should inhibit three components of the disease: tissue fibrosis, vascular abnormalities and autoimmunity. Moreover, potential drug needs to be considered in the context of specific four subsets of the disease: fibroproliferative, inflammatory, limited, and normal-like. It was shown that various subsets could have different profile of specific cytokines: TGF $\beta$ - associated with fibroproliferative and inflammatory type of disease and IL13 - mediator of fibrotic and vascular pathology.

Objectives: The aim of the study was to assess the level of TGF $\beta$ and IL-13 in SSc pts with various treatment regimen comparing to healthy control.

Methods: 55 patients ( $71 \%$ were women) with SSc diagnosed according to EULAR/ACR 2013 criteria were divided into 5 treatment groups: 1 st group -8 pts treated with methotrexate (MTX), 2-13 pts with mycophenolate mofetil (MMF), 35 pts with cyclophosphamide (CYC), 4th group- 7 pts with azatioprine (AZA), 5th group- 22 pts without immunosupressive treatment. All patients have been treated based on a scheme required for organs involvement in accordance with the update of EULAR recommendations from 2016. Mean age of patients was 54.56 \pm 13.97 . The blood and serum samples have been collected for basic examination. TGF $\beta$ and IL13 concentration in serum was quantitated by ELISA. Differences in cytokine concentration were determined using non-parametric Kruskal-Wallis test. The level of statistical significance was set at $p<0.05$. The modified Rodnan Skin Score (mRSS) examination were taken by one assessor at the beginning of the study and six months later. There were measured DLCO, HRCT, echocardiography and capillaroscopy.

Results: In $82 \%$ pts positive antinuclear antibodies have been revealed: in $16 \%$ positive results for CENPB were obtained and in 44\% for Scl70. Capillaroscopy shown in $23 \%$ of pts early pattern, in $38 \%$ - active pattern, and in $27 \%$ - late pattern. $32 \%$ of SSc pts had confirmed pulmonary fibrosis, while $90 \%$ - Raynaud's syndrome. The median of mRSS in CENPB (+) pts was $5,{ }^{4-24}$ in Scl70 (+) pts $12 .^{2-30}$ Statistically significant differences were found between IL13 and TGF $\beta$ levels in patients treated with immunosuppressants and healthy subjects. There was no correlation between IL13 or TGF $\beta$ with lung fibrosis progression or skin involvement.
Abstract AB0775 - Table 1. Comparison of serum level of IL 13 and TGFB in SSc pts

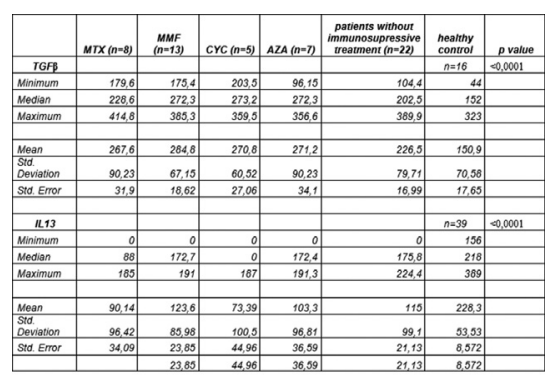

Conclusions: In conclusion, our findings indicate that IL 13 and TGF $\beta$ are characteristic cytokines in scleroderma, but these parameters did not correlate with severely progressive course of SSc.

Disclosure of Interest: None declared

DOI: 10.1136/annrheumdis-2018-eular.6049

\section{AB0776 MUSCLE ULTRASONOGRAPHY: A POTENTIAL NEW DIAGNOSTIC TOOL FOR INFLAMMATORY MYOPATHIES}

K. Bhansing ${ }^{1}$, C. Saris ${ }^{2}$, J. Fransen ${ }^{1}$, F. van den Hoogen ${ }^{1}$, A. Verrips ${ }^{3}$, R. Cooper ${ }^{4}$, P. van Riel ${ }^{5}$, B. van Engelen ${ }^{2}$, S. Pillen ${ }^{6}$, M. Vonk ${ }^{1}$. ${ }^{1}$ Rheumatology; ${ }^{2}$ Neurology, Radboud University Medical Center, ${ }^{3}$ Neurology, Canisius Wilhemina Hospital, Nijmegen, Netherlands; ${ }^{4}$ Institute of Ageing and Chronic Disease, University of Liverpool, Liverpool, UK; ${ }^{5}$ Scientific Institute for Quality of Healthcare, Radboud University Medical Center, Nijmegen; ${ }^{6}$ Child Development and Exercise Center, University Medical Center Utrecht, Utrecht, Netherlands

Background: Quantitative muscle ultrasound (QMUS) imaging has proven to be a useful, non-invasive technique to visualise normal and pathological skeletal muscle tissue. ${ }^{1}$ Electromyography (EMG) findings are not always disease specific in patients suspected of idopathic inflammatory myopathies (IIM).

Objectives: To assess diagnostic value of QMUS in patients suspected for an IIM and to compare results with EMG.

Methods: In 57 patients, suspected for IIM, panel diagnosis blinded for QMUS was used as reference standard. QMUS results were used to classify patients according an ultrasound neuromuscular disorder (NMD) algorithm (normal/borderline/abnormal). The predictive value of QMUS and EMG was assessed in a two by two table and a multivariate logistic regression model.

Results: Twenty-two patients (39\%) were diagnosed with IIM; 8 polymyositis, 4 dermatomyositis, 4 necrotizing myopathy, 3 inclusion body myositis and 3 nonspecific myositis. Sixteen patients were classified with other NMD. We found an increased echointensity of the sternocleidomastoid, biceps, forearm flexor and tibialis anterior in the IIM group. Sensitivity, specificity, positive and negative predictive values (PPV/NPV) were $82 \%, 51 \%, 51 \%, 82 \%$ for ultrasound NMD algorithm and $63 \%, 64 \%, 50 \%, 75 \%$ for EMG. Multivariate analyses showed area under the curve (AUC) (0.81) (0.69-0.92) for ultrasound NMD algorithm, EMG (0.79) (0.67-0.92) and ultrasound NMD algorithm plus EMG (0.82) (0.70-0.93).

\begin{tabular}{lccc}
\hline Predictor & Model A & Model B & Model C \\
\hline Age $\geq 50$ year & $2.97(0.65-$ & $2.88(0.62-$ & $2.98(0.62-$ \\
Serum $C K \geq 2 \times$ upper limit & $13.59)$ & $13.28)$ & $14.21)$ \\
& $7.52(1.57-$ & $8.76(1.55-$ & $7.35(1.22-$ \\
& $36)$ & $49.55)$ & $44.21)$
\end{tabular}

Muscle ultrasound

Total echointensity of proximal muscles/

measured muscle

Total number of affected proximal muscles

Distal muscles affected (yes/no)

NMD algorithm:

No NMD

-Borderline presence of NMD (yes/no)

Reference:

$1.61(0.13$ 18.87)

-Presence of NMD (yes/no)

$3.01(0.44$

EMG qualitative report:

20.55)

Negative myopathic results

Borderline myopathic results

Positive myopathic results

Cox and Snell $R$ square

Nagelkerke R square

Hosmer Lemeshow Test

AUC $(95 \% \mathrm{Cl})$ 